\title{
Application Value of Transvaginal Ultrasound on Early Screening for Ectopic Pregnancy
}

\author{
Chen Yu, Chunxue Xu, Jun Huang* \\ Ultrasonic Department, The First Affiliated Hospital of Jinan University, Guangzhou 510630, Guangdong Province, China
}

\begin{abstract}
[Abstract] Objective: To explore the effect and value of transvaginal ultrasound in screening early ectopic pregnancy.
Methods: A total of 146 patients with ectopic pregnancy in our hospital were selected. The patients' entry time was from May 2018 to December 2020. 146 patients were divided into two groups. The control group was screened by abdominal ultrasound with 73 cases, and the experimental group was screened by vaginal ultrasound with 73 cases. The uterine adnexal mass, pelvic effusion, peritoneal effusion and diagnostic accuracy were detected. Results: The positive number of abdominal ultrasound was significantly higher than that of vaginal ultrasound; In the detection process of abdominal ultrasound, 54 cases of ectopic pregnancy, 19 cases of non-ectopic pregnancy, the detection rate was 73.97\%, 26 cases of ectopic pregnancy, 47 cases of non-ectopic pregnancy, the detection rate was $32.62 \%$. Conclusion: The accuracy of transvaginal ultrasound is relatively high, and the accuracy of ectopic pregnancy examined by transvaginal ultrasound is higher, which is more friendly to patients, less traumatic and high operability. It can provide more accurate reports for patients and relieve the psychological pressure of patients, which is worthy of application in actual screening.
\end{abstract}

Key words: Ultrasound; Early screening; Ectopic pregnancy

Publication date: May, 2021; Publication online: 31 May, 2021

*Corresponding author: Jun Huang, $\underline{\text { 845298945@qq.com }}$

\section{Introduction}

Ectopic pregnancy is a common disease in gynecology. It may be caused by systemic inflammation or the implantation of the pregnant egg outside the uterus. It is thought that ectopic pregnancy is similar to other diseases, so it is easy to cause other diseases to be misdiagnosed as ectopic pregnancy. Ectopic pregnancy is misdiagnosed as other diseases. In other unexpected locations of the uterus, it does not have the conditions for the development of the pregnant egg. If the implantation is carried out outside the uterus, it is easy to cause life-threatening to the patients, so it needs timely medical treatment, examination and treatment. The safety of ultrasound diagnosis is high, the operability is strong, and the trauma to the patients is also small, which is worthy of promotion in clinical practice. This paper compares the diagnostic results of vaginal ultrasound and abdominal ultrasound, and compares the value of the two kinds of ultrasound It is reported as follows.

\section{Data and methods}

\subsection{General information}

Methods: a total of 146 patients with ectopic pregnancy in our hospital were selected and entered from May 2018 to December 2020. According to the random number table method, 146 patients were divided into two groups. The control group was screened by abdominal ultrasound, the number of cases was 73, the age was between 21 and 32 years old, the average age was $(27.8 \pm 2.1)$; The experimental group used transvaginal ultrasound screening, the number of cases was 73, the age was between 22-31 years old, the average age was (27.6 \pm 1.9$)$, the entry standard was patients with ectopic pregnancy phenomenon, for patients with other gynecological diseases were excluded, the study has been approved by the ethics committee, general information does not affect the experimental results, $\mathrm{t}$ value was 0.6034 , $\mathrm{P}$ value was 0.5472 .

\subsection{Methods}

The ultrasonic diagnostic instruments used in the study are 
the same ${ }^{[1]}$. Abdominal ultrasound: Before the examination, the patient needs to be informed of the behavior of holding urine, so as to ensure the clear imaging during the examination and avoid the impact of this behavior on the diagnostic scanning results. Set the frequency of the instrument probe between $3 \sim 5 \mathrm{MHz}$. The patient lies on his back and scans the pubic symphysis and below in multiple sections. Carefully observe the uterine appendages, gestational sac, mass, uterine size, endometrial thickness, etc.

Transvaginal ultrasound (TVS): During the process of transvaginal ultrasound, patients need to adopt proper posture and strictly disinfect the vulva to avoid infection caused by examination and destroy the bacterial flora in the vagina. Besides, the probe needs to be disinfected. After applying couplant, the probe needs to be put on a condom. The probe was slowly inserted into the patient's vagina for scanning, and uterine adnexa, pregnancy sac, mass, uterine size, endometrial thickness, etc., were carefully observed. The frequency of the probe should be set at $8 \mathrm{MHz}$.

\subsection{Observation indexes}

The diagnostic accuracy of uterine adnexal mass, pelvic effusion, peritoneal effusion, and statistical analysis.

\subsection{Statistical methods}

SPSS 25.0 software was used to analyze the data. The measurement data were expressed by the mean " $\mathrm{x} \pm \mathrm{s}$ " standard deviation, and the comparison between groups was performed by $t$ test. The counting data were analyzed by $\chi^{2}$ test. The difference was statistically significant $(P<0.05)$.

\section{Results}

\subsection{Comparison of the positive rate of ultrasound images between the two groups}

In the ultrasonic image diagnosis of abdominal ultrasound, there were 19 cases of uterine adnexal mass, 21 cases of ascites, and 18 cases of pelvic effusion. In the ultrasonic image of vaginal ultrasound, there were 9 cases of uterine adnexal mass, 7 cases of ascites, and 7 cases of pelvic effusion. Among them, the $\chi^{2}$ of uterine adnexal mass was 4.4189 , the $\chi^{2}$ of ascites was 8.6610 , and the $\chi^{2}$ of pelvic effusion was 5.8400 , all $\mathrm{P}<0.05$ The positive detection number of abdominal ultrasound was significantly higher than that of vaginal ultrasound, as shown in Table 1.

Table 1. Comparison of the positive rate of ultrasound images between the two groups [n (\%)]

\begin{tabular}{ccccc}
\hline Group & Number of cases & Adnexal mass of uterus & Pelvic effusion & Ascites \\
\hline experience group & 73 & $9(12.33)$ & $7(9.59)$ & $7(9.59)$ \\
control group & 73 & $19(26.03)$ & $21(28.77)$ & $18(24.66)$ \\
$\chi^{2}$ & & 4.4189 & 8.6610 & 5.8400 \\
$P$ & & & $<0.05$ & \\
\hline
\end{tabular}

3.2 Comparison of the detection rate of ectopic pregnancy by two ultrasound diagnostic methods

In the detection process of abdominal ultrasound, 54 cases of ectopic pregnancy, 19 cases of non ectopic pregnancy, the detection rate was $73.97 \%, 26$ cases of ectopic pregnancy, 47 cases of non ectopic pregnancy, the detection rate was $32.62 \%$, of which $\chi^{2}=21.6788$, the difference was statistically significant $(P<0.05)$, as shown in Table 2 .

Table 2. Comparison of the detection rate of ectopic pregnancy by two ultrasound diagnostic methods

\begin{tabular}{ccccc}
\hline Group & Number of cases & Ectopic pregnancy & Non ectopic pregnancy & Detection rate [n (\%)] \\
\hline experience group & 73 & 26 & 47 & $26(35.62)$ \\
control group & 73 & 54 & 19 & $18(73.97)$ \\
$\chi^{2}$ & & & 21.6788 \\
$P$ & & & $<0.05$ \\
\hline
\end{tabular}

\section{Discussion}

Ectopic pregnancy, also known as "ectopic pregnancy", mainly refers to the implantation and development of fertilized eggs in extra uterine organs or tissues. In the diagnosis, when ectopic pregnancy is diagnosed, it is mostly tubal pregnancy, which accounts for the largest proportion of ectopic pregnancy, accounting for nearly $95 \%$. Inflammation 
inside or around the fallopian tube makes the fertilized egg move and implant in the fallopian tube, or abnormal estrogen and progesterone interfere with the normal function of the pregnant egg. Because the number of early ectopic pregnancy is relatively small and the clinical symptoms are not clear, it is easy to miss or misdiagnose ${ }^{[2]}$. Although the diagnosis efficiency of early ectopic pregnancy has been greatly improved due to the latest development of clinical diagnosis technology, especially the popularity of ultrasound diagnosis, in the past, the diagnosis of early pregnancy was mainly composed of transabdominal ultrasound. In the process of screening, abdominal ultrasound is easily affected by the patients themselves, such as obesity, flatulence, abdominal wall scar, abdominal wall tension and bladder filling. It has a certain impact on imaging and is easy to cause misdiagnosis. Compared with abdominal ultrasound, transvaginal ultrasound has higher ultrasound frequency, clearer imaging, larger scanning range and better resolution, which can better check and diagnose the cause of patients. The sensitivity and flexibility of transvaginal ultrasound detection are relatively high, which has obvious effect on the diagnosis of ectopic pregnancy. In clinical practice, doctors advocate the application of transvaginal ultrasound to diagnose ectopic pregnancy. The etiology of the patients was detected and diagnosed. In this clinical study, transvaginal ultrasound has high accuracy in the diagnosis of ectopic pregnancy, and has the characteristics of simple operation and non-invasive, which has important application value in clinical practice. In the application of transvaginal ultrasound in the diagnosis of ectopic pregnancy, we should pay attention to the following aspects to better ensure the diagnostic efficiency

First of all, we should pay attention to the judgment of true and false pregnancy pocket. During pregnancy, oval or spindle shaped non echo pseudopregnancy bags may appear in the uterine cavity due to changes in hormone levels. This is the core of diagnosis combined with the past. The author thinks that the true and false bags can be distinguished in the following three cases. The upper pouch is located in the uterine cavity and surrounded by the endometrium, while the actual pouch is located in the endometrium and the endometrium is located outside the uterine cavity. "Signal, that is, there is a hypoechoic ring around the echo ring, and the actual pregnancy bag will have this symptom. At the same time, it can be clearly observed that during the vertical scanning, the pregnancy bag and the cervical canal cannot communicate." Gastric gestational sac is caused by abnormal pregnancy, which is often accompanied by endometrial and decidual reactions. At the same time, there are slight bleeding symptoms due to intrauterine bleeding. The actual blood flow sac before and after pregnancy mainly comes from the trophoblastic blood flow artery of the primordial placenta. In the process of examination, the above characteristics can be combined to determine whether the patient's gestational cyst is true or false ${ }^{[3-6]}$.

Second, the possibility of ectopic pregnancy in the ovary, abdominal cavity and cervix is only 5\%. Most patients have tubal pregnancy. According to the ultrasonic examination stage, tubal ectopic pregnancy can be divided into non rupture period. First of all, in the non rupture stage, the ultrasound image shows that the patient's appendix can see the complete fetal sac, indicating that there is a strong echo shedding around it. At the same time, in more common patients, we can see the beating of yolk sac and primitive heart tube. In this case and around the gestational bag, more abundant semicircular blood flow signals can be seen. This is the trophoblastic blood flow. The RI ranged from 0.4 to 0.55 . However, the blood flow impedance of patients with ovarian cancer is low, so it is necessary to distinguish ectopic pregnancy from ovarian cancer in the diagnosis of ectopic pregnancy. Most of the ovarian malignant tumors are scattered in the tumor, which can be diagnosed with CDFI. When the tubal pregnancy is in the period of abortion, the ultrasound image shows mixed quality and no echo area, because the echo area is surrounded by thick and strong echo ${ }^{[7]}$. However, ultrasound imaging of tubal ectopic pregnancy during abortion is more complex than that during unruptured period. For example, in the early stage of rupture, patients show echo disturbance and echo disturbance with increased echo of abnormal quality, and as the rupture continues to increase, the echo of abnormal packaging quality increases. When the gestational sac completely ruptured, due to repeated bleeding symptoms, ultrasound showed uneven, chaotic mass. At the same time, in the area around the uterus, you can see obvious fluid dark areas caused by the patient's pelvic and abdominal bleeding.

Third, in cesarean section, uterine scar, corneal pregnancy, cervical pregnancy, pregnancy can be confirmed, and in each pregnancy, pregnancy bag can be clearly observed near the fundus of uterus. The cross-sectional image shows that the patient's uterine horn is asymmetric, and the pregnant pouch can be seen at the same time ${ }^{[8-11]}$. 
Ultrasound images of cervical pregnancy usually show that the uterus is flask shaped, and the normal size of the uterine body increases at the top and at the bottom of the cervix. The blood flow signal is ri0.4 and in the uterus. The orifice is closed tightly and the original beating of the heart tube can be seen. Ultrasound images of cesarean scar pregnancy will show that the patient's gestational sac has been implanted into the lower anterior wall of the uterus, resulting in obvious scar. The echo of the embryo placenta is strong, and the inner wall of the cervix is closed. From the inside, the pregnant bag embryo or the unprocessed heart tube can be seen.

To sum up, the accuracy of transvaginal ultrasound is relatively high, the accuracy of ectopic pregnancy examined by transvaginal ultrasound is higher, it is more friendly to patients, less traumatic and high operability, it can provide more accurate reports for patients, relieve the psychological pressure of patients, it is worthy of application in the actual screening.

\section{References}

[1] Cao HX. Clinical application value of transvaginal color Doppler ultrasound combined with serum IL-37 and SCCA in the diagnosis of cervical cancer $[\mathrm{J}]$. Contemporary Medicine, 2021,27 (08): 14-17.

[2] Yi BXa, Xu HX, Xu P. Application value of intrauterine cytology combined with transvaginal ultrasound in screening of endometrial cancer $[\mathrm{J}]$. Chinese Journal of Obstetrics and Gynecology, 2021,22 (02): 181-182.

[3] Liu ZZ, Wei Y, Deng S, Li JC. The value of transvaginal real-time three-dimensional hysterosalpingography in evaluating tubal patency $[\mathrm{J}]$. Journal of Reproductive Medicine, 2021,30 (03):
$362-366$

[4] Wang R. Application value and imaging characteristics of transvaginal ultrasound in the diagnosis of endometrial lesions [J]. Medical Equipment, 2021,34 (05): 34-35.

[5] Li YY, Song DY. Clinical value of transabdominal ultrasound combined with transvaginal ultrasound in diagnosis of adenomyosis $[\mathrm{J}]$. Chinese Journal of Modern Drug Application, 2021,15 (05): 61-63.

[6] Chai X. Characteristics and diagnostic value of transvaginal three-dimensional ultrasound in endometrial polyps [J]. Sichuan Journal of Anatomy, 2021,29 (01): 159-160.

[7] Han H, Liu QQ. Comparison of transabdominal and transvaginal color Doppler ultrasound in the diagnosis of adenomyosis and analysis of risk factors of adenomyosis [J]. China Maternal and Child Health, 2021,36 (06): 1443-1445.

[8] Peng XM, Li SJ, Li S, Zhou FY. Diagnostic value of transvaginal high frequency ultrasound in preoperative evaluation of endometrial cancer staging and lymph node metastasis [J]. Oncology Imaging, 2021,30 (01): 36-42.

[9] Mao W. Cervical chlamydia screening before induced abortion and its clinical significance $[\mathrm{J}]$. Inner Mongolia Traditional Chinese Medicine, 2014,33(21): 60-61.

[10] Zhu M, Ma YH, Qin LX, Ma JX, Zhao XY, Chen W. Analysis of clinical value of B-ultrasound screening in 8-12 $(+6)$ weeks of early pregnancy $[\mathrm{J}]$. Journal of Kunming Medical University, 2012, 33(12): 84-86.

[11] Li NM, Yang WG. Value of transvaginal ultrasound in early diagnosis of ectopic pregnancy $[\mathrm{J}]$. China Maternal and Child Health Care, 2010, 25(5): 717-718. 